\title{
SYMMETRY CONSIDERATIONS IN CLOSED FIRST-ORDER KINETIC SYSTEMS
}

\author{
Erik K. GRIMMELMANN and Lawrence L. LOIIR Jr. \\ Department of Chemistry, The University of Michigan, \\ Ann Arbor, Michigan 48109, USA
}

Received 8 August 1977

Revised manuscript received 3 November 1977

\begin{abstract}
The analysis of a highly symmetric type of exactly lumpable, closed systen of coupled, first-order rate equations shows that complete information about the equilibrium solution and all modes of relaxation is calculable from matrices with dimensions less than the number of states in the system.
\end{abstract}

\section{Introduction}

Systems of coupled first-order chemical reactions, of ten referred to as monomolecular reaction systems, are of considerable interest. For example, some real chemical systems are of this type, and the kinetics of many more can be approximated by first-order rate equations, a technique referred to as linearization [1] . In the limit of small perturbations from thermody. namic equilibrium, the relaxation back to equilibrium of all reversible, closed chemical systems obeys firstorder kinetics [2-5]. Biologists often model the flow of tracers from compartment to compartment of multicompartment systems using first-order kinetics $[6,7]$. In addition, these reaction schemes are mathematically simple, at least compared to systems of reactions involving higher-order kinetics, and elegant methods have been developed for treating them. Much has been proven about the solutions of the coupled differential equations which represent these systems.

The formalism concerned with the existence, uniqueness, and stability of steady-state and periodic solutions of sets of coupled differential equations is often referred to as dynamical systems theory [8] Closed, monomolecular reaction systems are represented by the simplest of such sets of equations, those consisting of homogencous, first-order linear differential equations with constant coefficients. A system is said to be closed if there are neither sources nor sinks for any species. In these sets of differential equations the concentration of each species is represented by a continuous variable whose time evolution is fully determined by the rate constants and the initial conditions. This is in contrast to a stochastic model in which continuous concentration variables are replaced by discrete random variables and rate constants by transition probabilities [9-11]. It has been shown that in closed linear systems the means of the discrete random concentration variables obey the deterministıc differential equations for the continuous concentrations [11] . Thus, the results we derive in the present work are of relevance also to closed linear systems to which a stochastic model is applicable.

In section 2 we review the mitrix formulation of sets of linear, first-order differential equations [3-7, 12-14] and the lumping of individual states into sets of states $[15,16]$. In section 3 we analy $2 c$ a specific highly symmetric type of exactly lumpable [15] system and show that by using symmetry considerations complete information about any mode of relaxation of the system is calculable without having to calculate the complete solution. We also employ group theory in classifying the modes of relaxation for certain cases.

\section{Matrix formulation and lumping}

The matrix formulation of sets of coupled, homoge- 
neous, linear, first-order differential equations with constant coefficients $[3-7,12-14]$ is well known. The reader is referred to Wei and P'rater [14] for further details and numerous examples, although our notation differs somewhat from theirs. We consider a closed system cunsisting of $n$ states labeled $1, \ldots, n$. Let $x_{i}(t)$ be the fractional concentration (mole fraction) of species in state $i$ at time $t$, and let $w_{i j}$ be the time independent first-order rate constant for the transition from state $i$ to state $j$. The time evolution of each $x_{i}(t)$ can then be represented by a master equation,

$\dot{x}_{j}(t)=\sum_{j}\left[w_{j i} x_{j}(t)-w_{i j} x_{i}(t)\right]$

The set of $n$ such equations can be placed into matrix form,

$\dot{X}(t)=\mathbf{A X}(t)$,

where $\dot{X}(t)$ and $X(t)$ are $n$-dimensional column vectors whose clements are $\dot{x}_{i}(t)$ and $x_{i}(t)$ respectively and $A$ is an $n \times n$ matrix whose elements are

$a_{z j}=w_{j i} \quad$ for $i \neq j$,

$a_{i j}=-\sum_{k \neq i} w_{i k}$.

If $A$ can be diagonalized, the solution of (1) corresponding to the $j$ th eigenvalue $\lambda_{j}$ of $A$ is

$x_{i}^{(j)}(t)=v_{i}^{(j)} \exp \left(\lambda_{j} t\right)$,

where $v_{i}^{(j)}$ is the $i$ th clement of the eigenvector corresponding to the $j$ th eigenvalue. The general solutions of (1) are linear combinations of the $x_{i}^{(j)}(t)$,

$x_{i}(t)=\sum_{j} b_{j} x_{i}^{(j)}(t)$

where the $b_{j}$ are determined by the initial conditions.

An equilibrium solution (4) of (1) is one for which $\lambda_{j}=0$. Zero is always an eigenvalue of $A$, and if $A$ is an irreducible matrix, it is a simple cigenvalue [6]. In the present work we assume that the $A$ matrices for systems of interest are irreducible, so that we are considering only systems having unique equilibrium solutions.

In general, the eigenvalues of $A$ are complex, but the real part of each eigenvalue must be non-positive [13] . However, the requirement of detailed balance imposes
$[6,13]$ relationships among the $w_{i j}$ such that all eigenvalues are real. We consider only systems which have solutions of the form (5) and only one eigenvalue (labeled $\lambda_{1}$ ) of zero. The initial conditions can be thought of as a displacement from equilibrium in which each coefficient $b_{i}(j>1)$ is a measure of the displacement in the $j$ th mode of the system. As time progresses the system approaches its unique equilibrium as the displacement from equilibrium in the $j$ th mode decays as $\exp (\lambda, t)$.

It is often convenient to group individual states into sets of states and consider the kinetic equations only for such sets rather than for the individual states within each set. For first-order systems such groupings are most useful when the concentration of each set, which is the sum of the concentrations of its component states, itself follows first-order kinetics. Wei and Kuo have called this grouping "lumping" and have presented a formalism for it $[15,16]$.

We consider groupings of $n$ states into $\bar{n}$ sets where each state is contained in exactly one set; Wei and Kuo have termed such groupings "proper lumpings". We define the identity lumping as that which places each state in its own set; for this lumping $n=\hat{n}$, while for all others $n>\hat{n}$. Greek letters will be used as durnmy indices for sets of states; thus, let $\hat{x}_{\alpha}(t)$ be the fractional concentration in set $\alpha$ at time $t$,

$\tilde{x}_{\alpha}(t)=\sum_{i \in \alpha} x_{i}(t)$

where the sum is over all states in set $\alpha$. The $\hat{x}_{\alpha}(t)$ may be viewed as the elements of an $\dot{n}$-dimensional column vector $\hat{X}(t)$ and the transformation of $X(t)$ into $\hat{X}(t)$ may be accomplished by means of an $\hat{n} X n$ lumping matrix $M$ [15],

$\hat{X}(t)=\mathbf{M X}(t)$.

The elements $m_{\alpha j}$ of $M$ are 1 if $j \in \alpha$ and 0 otherwise. The lumping defined by $M$ is exact if and only if there exists an $\bar{n} \times \bar{n}$ matrix $A$ such that

$\hat{\bar{X}}(t)=M \hat{X}(t)=\hat{A} \hat{X}(t)$

describes the kinetic behavior of the lumped system [15]. We may define $\hat{\mathbf{A}}$ as

$\hat{\mathbf{A}} \equiv$ MAM $^{-1}$,

where $M^{-1}$ is a right inverse of $M ; M^{-1}$ is an $n \times \dot{n}$ 
matrix whose elements $m_{j \alpha}^{-1}$ may be chosen as $1 / n_{\alpha}$ if $j \in \alpha$ and 0 otherwise, where $n_{\alpha}$ is the number of states in set $\alpha$. A necessary and sufficient condition [15] for Á to satisfy (8) is

MA $=\hat{\text { ÀM. }}$.

Eq. (10) does not necessarily follow from (9) since $M^{-1}$ is a left inverse of $M$ only for the identity lumping. For (8) and (10) to hold, there must exist a set of $k_{\alpha \beta}$ for all $\alpha, \beta$ with $\alpha \neq \beta$, such that [15]

$\sum_{j \in \beta} w_{i j}=k_{\alpha \beta} \quad$ for all $i \in \alpha$.

\section{Symmetry considerations}

\subsection{Equivalent interset transitions}

We now consider a highly symmetric, specific type of exactly lumpable system. We assume that there exists a set of $w_{\alpha \beta}$ for all $\alpha, \beta$ with $\alpha \neq \beta$, such that

$w_{i j}=w_{\alpha \beta} \quad$ if $i \in \alpha$ and $j \in \beta$.

These rate constants trivially satisfy (11) with $k_{\alpha \beta}=$ $n_{\beta} w_{\alpha \beta}$. For convenience we arrange the $n$ state labels so that the states in set 1 are numbered $1, \ldots, n_{1}$; those in set $2, n_{1}+1, \ldots, n_{1}+n_{2}$; etc. The states in set $\alpha$ are alternatively designated $1_{\alpha}, 2_{\alpha}, \ldots, n_{\alpha}$.

We can write $M$ in partitioned form where each $M_{\alpha \beta}$ is a $1 \times n_{\alpha}$ matrix whose elements are 1 if $\alpha=\beta$ and 0 otherwise. Similarly, $M^{-1}$ can be written in partitioned form where each $M_{\alpha \beta}^{-1}$ is an $n_{\alpha} \times 1$ matrix whose elements are $1 / n_{\alpha}$ if $\alpha=\beta$ and 0 otherwise. The matrix $A$ can be partitioned also. The diagonal blocks are $n_{\alpha} \times n_{\alpha}$ matrices, $A_{\alpha \alpha}$, whose elements are

$a_{i j}=w_{j_{\alpha} i_{\alpha}}$ if $i \neq j$,

$a_{i i}=-\left[K_{\alpha}+\sum_{\substack{k \neq i_{\alpha} \\ k \in \alpha}} w_{i_{\alpha} k}\right]$,

where

$K_{\alpha}=\sum_{\beta \neq \alpha} k_{\alpha \beta}$

If $n_{\alpha}=1$, then $A_{\alpha \alpha}$ is the scalar element $-K_{\alpha}$. The off- diagonal blocks of $A$ are $n_{\alpha} \times n_{\beta}$ matrices, $A_{\alpha \beta}$, whose elements are all $w_{\beta \alpha}$. The lumped matrix $\bar{A}$ is defined by (9) and its elements may be written in terms of the partitions of $M, A$, and $M^{-1}$,

$\hat{A}_{\alpha \beta}=\mathbf{M}_{\alpha \alpha} \mathbf{A}_{\alpha \beta} \mathbf{M}_{\beta \beta}^{-1}$,

which leads to

$\hat{A}_{\alpha \beta}=k_{\beta \alpha} \quad$ if $\alpha \neq \beta$,

$\hat{A}_{\alpha \alpha}=-K_{\alpha}$.

We now examine the relationship between the eigenvalues and eigenvectors of $\hat{A}$ and those of $A$. The eigenvector of $A$ corresponding to the eigenvalue $\lambda_{j}$ can be written in partitioned form,

$V^{(j)}=\left(\begin{array}{l}v_{1}^{(j)} \\ v_{2}^{(j)} \\ \vdots \\ v_{i}^{(j)}\end{array}\right)$

where $V_{\alpha}^{(l)}$ is an $n$-dimensional column vector whose elements are $V_{1_{\alpha}}^{(j)}, V_{2}^{(j)}, \ldots, V_{n_{\alpha}}^{(j)} . \bar{V}^{(j)}$ is defined by

$\hat{V}^{(j)}=\mathbf{M} \boldsymbol{V}^{(j)}$,

and thus the elements of $\tilde{V}^{(j)}$ are

$\hat{V}_{\alpha}^{(j)}=M_{\alpha \alpha} V_{\alpha}^{(j)}=\sum_{i \in \alpha} V_{i}^{(j)}$

Wei and Kuo [15] and Ozawa [17] have shown that $\hat{V}^{(j)}$ is either an eigenvector of $A$ with eigenvalue $\lambda_{j}$ or a vector with all zero elements. In the former case, $V^{(j)}$ is the equilibrium solution or one of the modes of interest relaxation, and in the latter case, $V^{(j)}$ is one of the modes of intraset relaxation.

The eigenvalues of $\hat{\mathbf{A}}$ are also the eigenvalues for the equilibrium solution and the interset modes of $A$, and the elements of the eigenvectors of $\hat{A}$ are the sums of groups of elements of the corresponding eigenvectors of $A$. We now consider the individual elements of an equilibrium or interset cigenvector of A. Let $V^{(j)}$ be such an eigenvector with $\lambda_{j}$ as its eigenvalue. The partitions of $V^{(j)}$ obey the equations,

$\sum_{\beta} A_{\alpha \beta} V_{\beta}^{(j)}=\lambda_{j} V_{\alpha}^{(j)}$, for all $\alpha$. 
For a particular partition, for example that corresponding to set $\alpha$, we can separate $V_{\alpha}^{(j)}$ from the other $V_{\beta}^{(j)}$,

$$
\mathbf{A}_{\alpha \alpha} \nu_{\alpha}^{(j)}-\lambda_{j} V_{\alpha}^{(j)}=-\sum_{\beta \neq \alpha} \mathbf{A}_{\alpha \beta} V_{\beta}^{(j)}
$$

Making use of the fact that the $A_{\alpha \beta}$ on the rhs of (21) are matrices whose elements are all $w_{\beta \alpha}$ and of (19) and using the symbol $I$ for the unit matrix and $I$ for a vector whose clements are all ones, we can obtain from (21).

$$
\left(A_{\alpha \alpha}-\lambda_{j} \mid\right) V_{\alpha}^{(j)}=-\left[\sum_{\beta \neq \alpha} w_{\beta \alpha} \hat{V}_{\beta}^{(j)}\right] I .
$$

We thus have $n_{\alpha}$ simultaneous linear equations in $n_{\alpha}$ unknowns, the elements a $V_{\alpha}^{(j)}$. One additional equation, (19), could be used to eliminate one unknown.

We now consider the intraset modes of relaxation. There are $n-i n$ of these modes, $n_{\alpha}-1$ for each set $\alpha$. If $V^{(/)}$corresponds to relaxation within set $\alpha$, then it may be written in partitioned notation with $V_{\beta}^{(j)}=0$ for $\beta \neq \alpha$. The non-zero part, $V_{\alpha}^{(j)}$, is simply an eigenvector of the $n_{\alpha} \times n_{\alpha}$ matrix $A_{\alpha \alpha}$ with cigenvalue $\lambda_{j}$. Therefore, to obtain the intraset modes of relaxation for a set $\alpha$ we need only diagonalize $A_{\alpha \alpha}$. Such a diagonalization yields $r_{\alpha}$ eigenvalues (not all necessarily distinct) and $n_{\alpha}$ eigenvectors corresponding to the equilibrium solution and the $n_{\alpha}-1$ modes of intraset relaxation. These intraset modes involve transitions between states in set $\alpha$ and states in other sets, but only in such a way that the concentration of species in any state not within set $\alpha$ remains constant. The only effect that the states outside of set $\alpha$ have on the intraset relaxation is the lowerng of the intraset eigenvalues by $K_{\alpha}$.

\subsection{Equivalent intraset transitions and permutational symmetry}

So far we have made no assumptions about the intraset transition probabilitics in the systems we have considered. We now examine the special case in which (12) is assumed to extend to intra as well as interstate rate constants. That is, we assume that for one ci more sets, set $\alpha$ for example, there exists a $w_{\alpha \alpha}$ such that

$w_{i j}=w_{\alpha \alpha} \quad$ if $i, j \in \alpha$.
Consistent with our definition of the $k_{\alpha \beta}$ we define $k_{\alpha \alpha}$ as $n_{\alpha} w_{\alpha \alpha}$.

For the equilibrium and interset modes, the restriction (23) leads to each of the elements of $V_{\alpha}^{(j)}$ being equal to $\hat{v}_{\alpha}^{(j)} / n_{\alpha}$. The restriction (23) greatly simplifies the intraset modes as well. The elements of $A_{\alpha \alpha \alpha}$ become

$a_{i j}=w_{\alpha \alpha} \quad$ if $i \neq j$,

$a_{i i}=-\left[K_{\alpha}+\left(n_{\alpha}-1\right) w_{\alpha \alpha}\right]$.

Diagonalizing $A_{\alpha \alpha}$ is trivial and leads to an cigenvalue of 0 and $n_{\alpha}-1$ degenerate eigenvalues of $-\left(K_{\alpha}+k_{\alpha \alpha}\right)$ which correspond to intiaset modes of relaxation. The degeneracy in the eigenvalues gives us considerable ficxibility in choosing the corresponding eigenvectors. A convenient choice is the set of the $n_{\alpha}-1, n_{\alpha}$-dimensional vectors,

$$
\left(\begin{array}{c}
1 \\
-1 \\
0 \\
\vdots \\
0 \\
0
\end{array}\right),\left(\begin{array}{c}
0 \\
1 \\
-1 \\
\vdots \\
0 \\
0
\end{array}\right), \cdots,\left(\begin{array}{c}
0 \\
0 \\
0 \\
\vdots \\
1 \\
-1
\end{array}\right)
$$

The restriction (23) on the intraset rate constants for set $\alpha$ makes all of the states in set $\alpha$ equivalent, that is, the system must be invariant to all permutations of state labels of the states within set $\alpha$. These $\pi_{\alpha}$ ! permutations form the group $S_{n_{\alpha}}$, the symmetric group. The reader is referred to Hamermesh [18], whose notation we use, for a thorough discussion of this group and its representations. Each permutation in $S_{n_{\alpha}}$ may be classified by its cycle structure which is designated $\left(1^{\alpha} 2^{\beta} 3^{\gamma} \ldots\right)$ where $\alpha$ is the number of 1 -cycles, $\beta$ the number of 2-cycles, etc. A 2-cycle is a transposition, 3 cycles may be written as a product of 2 transpositions, and arr $n$-cycle as a product of $n-1$ transpositions. Each class of $S_{n_{\alpha}}$ is labeled by the cycle structure of its members and the irreducible representations of $S_{n_{\alpha}}$ are labeled in a somewhat similar notation.

We now determine the irreducible representations to which the eigenvectors of the system belong. Since the eigenvectors corresponding to the equilibrium solution and the interset modes of relaxation have simply the 
values $V_{\alpha}^{(J)} / n_{\alpha}$ as their entries in the rows corresponding to states in set $\alpha$, these eigenvectors are invariant to all of the permutations in $S_{n_{\alpha}}$ and thus belong to the totally symmetric representation of $S_{n_{\alpha}}$. This representation is labeled $\left(n_{\alpha}\right)$. The $n-\hat{n}-n_{\alpha}+1$ eigenvectors corresponding to intraset modes of relaxation for sets other than $\alpha$ have zeros in the rows corresponding to states in set $\alpha$, and thus these eigenvectors also belong to the totally symmetric representation $\left(n_{\alpha}\right)$. We now consider the $n_{\alpha}-1$ eigenvectors corresponding to intraset modes for set $\alpha$. The relevant rows of these eigenvectors are given by (25). By inspection, it can be seen that the character of the representation to which these eigenvectors belong is simply $\alpha-1$ for the class with cylce structurc $\left(1^{\alpha} 2^{\beta} 3^{\gamma} \ldots\right)$. But $\alpha-1$ is also the character for the representation $\left(n_{\alpha}-1,1\right)$. Thus, the $n_{\alpha}-1$ eigenvectors for the intraset modes for state $\alpha$ belong to the representation $\left(n_{\alpha}-1,1\right)$, while the remaining $n-n_{\alpha}+1$ eigenvectors of the system belong to the totally symmetric representation $\left(n_{\alpha}\right)$.

We now consider the case in which the intraset rate constants follow (23) for more than one set, say sets $\alpha, \beta, \ldots, \gamma$. In this case, the system is invariant to the permutations of the direct product group

$\boldsymbol{G}=S_{\boldsymbol{n}_{\boldsymbol{\alpha}}} \times S_{\boldsymbol{n}_{\beta}} \times \ldots \times S_{n_{\gamma}}$,

in which the permutations in each component group act only on the state labels within its set. The $n$ eigenvectors for the equilibrium solution and interset modes belong to the totally symmetric representation of $G$ which is given by the direct product of the totally symmetric representation of its component groups, $\left(n_{\alpha}\right) \times$ $\left(n_{\beta}\right) \times \ldots \times\left(n_{\gamma}\right)$. Similarly, the $n-1$ eigenvectors for the intraset modes for set $\alpha$ belong to the representation $\left(n_{\alpha}-1,1\right) \times\left(n_{\beta}\right) \times \ldots \times\left(n_{\gamma}\right)$, those for the intraset modes for set $\beta$ to the representation $\left(n_{\alpha}\right) \times$ $\left(n_{\beta}-1,1\right) \times \ldots \times\left(n_{\gamma}\right)$, etc

\section{Summary}

Complete information about the equilibrium solution or any intraset or interset relaxation mode for the specific highly symmetric type of exactly lumpable kinetic system characterized by equivalent interset rate constants is shown to be calculable without solving for the complete solution. By contrast the analysis by Wei and Kuo $[14,15]$ of more general lumpable sys- tems does net include intraset relaxation modes or reveal the contributions of individual states to the equilibrium solution or interset modes. For systems invariant to permu ations of state labels within sets in addition to possessing equivalent interset rate constants, the relaxation modes are particularly simple and can be classified using group theory.

The question then arises as to when one might expect to encounter systems even approximately having these characteristics. While the states $1, \ldots, n$ may refer to distinct chemical species in the usual sense, they may instead refer to individual quantum states of a system. For example the sets $\alpha$ and $\beta$ may be sets of vibrational (or rotational-vibrational) states associated with electronic states $\alpha$ and $\beta$ respectively, with the $w_{i j}=w_{\alpha \beta}$ for $i \in \alpha$ and $i \in \beta$ heing internal conversion (or intersystem crossing) rate constants. The intraset relaxations in such an ex.ımple refer to vibrational relaxations within a given clectronic state. Alternatively the sets $\alpha$ and $\beta$ may be sets of spin components for different electronic or nuclear spin states. We have recently used the preceding analysis in describing the kinetics of the equilibration between planar diamagnetic and tetrahedral pardmagnetic $\mathrm{Ni}(\mathrm{II})$ complexes $[19,20]$.

\section{References}

[1] W.E. Boyce and R.C. di Prima, Clementary differentiai equations and boundary value problems (Wlley, New York, 1969) ch. 9.

[2] G.H. Czerlinski, Chemical relaxatton (Decher, New York, 1966).

[3] M. Eigen and L. de Maeyer, in: Investigation of rutes and inechanisms of reactions, Part II, ed. G.G. Hammes, Techniques of chemistry, Vol. 6 Niley, New York, 1974) pp. $63-146$.

[4] K. Kust'n, D. Shear and D. Kleitman, J. Theoret. Biol. 9 (1965) 186.

[5] H.J.G. Ixayman, Isracl J. Chem. 11 (1973) 489.

[6] J.Z. Hearon, Ann. NY Acad. Sci. 108 (1963) 36.

[7] C.D. Thron, Bull. Math. Biophys. 34 (1972) 277.

[8] R. Rosen, Dynamical systems theory in biology, Vol. 1. Stability theory and its applications (Wley-Interscience, New York, 1970).

[9] A.J.F. Siegert; Phys. Rev. 76 (1948) 1708.

[10] L.M. Krieger and P.J. Gans, J. Chem. Phys. 32 (1960) 247.

[1 1 ] I.G. Darvey and P.J. Staff, J. Chem. Phys. 44 (1966) 990.

[12] B.J. Zwolinski and 11. Eyrng, J. Ain. Chem. Soc. 69 (1947) 2702. 
[13] J.Z. Hearon, Bull. Math. Biophys. 15 (1953) 121.

[14] J. Wei and C.D. Prater, Advan. Catalysis 13 (1962) 203.

[15] J.Wei and J.C.W. Kuo, Ind. Eng- Chem. Fund. 8 (1969) 114.

[16] J.C.W. Kuo and J. Wei, Ind. Eng. Chem. Fund. 8 (1969) 124.

[17] Y. Ozawa, Ind. Eng. Chem. Fund. 12 (1973) 191.
[18] M. Hamermesh, Group theory and its applications to physical problems (Addison-Wesley, Reading, 1962) ch. 7.

[19] L.L. Lohr Jr. J. Am. Chem. Soc., to be published.

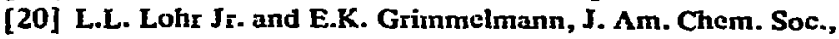
to be published. 